\title{
Influencia de la calidad de vida, la participación y la resiliencia en el desarrollo de alumnos de educación primaria
}

\author{
Influence of quality of life, participation and resilience on the \\ development of primary education students
}

\begin{abstract}
Resumen
El objetivo de esta investigación

fue evaluar la calidad de vida,

la participación en actividades

extraescolares y las características

resilientes del alumnado de primaria,

e identificar factores contextuales

que limitan o facilitan su desarrollo

saludable. Participaron I 56

alumnos, de entre го у I 3 años, de las comunidades autónomas de

Castilla y León y Andalucía. Ochenta presentaban necesidades específicas de apoyo educativo (NEAE). Los instrumentos utilizados fueron el Cuestionario de calidad de vida KIDSCREEN-27, el Cuestionario de Participación en Actividades Extraescolares CAPE/PAC y la versión traducida y adaptada al castellano del Cuestionario de Resiliencia CYRM. Los participantes obtuvieron puntuaciones elevadas en calidad de vida y resiliencia, y moderadas en participación en actividades extraescolares. El alumnado con NEAE obtuvo puntuaciones inferiores en la percepción de su bienestar emocional, recursos resilientes y participación en actividades de tipo físico. Estos resultados constituyen un punto de partida para elaborar un marco de indicadores basado en los modelos de inclusión y calidad de vida.
\end{abstract}

\section{Palabras clave:}

Calidad de vida, participación, resiliencia, alumnado de primaria.

\begin{abstract}
The aims of this research were to assess the quality of life, participation in leisure activities and resilience of primary school students, and to identify personal and environmental factors that limit or enhance their wellbeing and positive development. I 56 primary students from the autonomic communities of Castilla y León and Andalucía, aged between Io and I 3 years old, participated in the research. 80 of them had special educational needs (SEN). The instruments used were Quality of Life questionnaire KIDSCREEN-27, Children's Assessment of Participation, Enjoyment and Preference CAPE/PAC, and a Spanish version of Child and Youth Resilience Measure CYRM. Participants had high scores on quality of life and resilience and moderate scores in participation in recreation and leisure activities. Students with SEN scored less than their peers on perception of emotional well-being, resilient resources and participation in physical activities. These results provide information to develop an indicators framework based on inclusion and quality of life of models.
\end{abstract}

\section{Keywords:}

Quality of life, participation, resilience, primary students.

\author{
Beatriz Ruiz Fernández \\ <b.rufe@usal.es> \\ Universidad de Salamanca \\ María Gómez Vela \\ <mgv@usal.es> \\ Universidad de Salamanca
}

\section{Ramón Fernández Pulido} <rfpulido@usal.es>

Universidad de Salamanca

\section{Marta Badia Corbella}

<badia@usal.es>

Universidad de Salamanca

Para citar:

Ruiz, B. et al. (20I7): "Influencia de la calidad de vida, la participación y la resiliencia en el desarrollo de alumnos de educación primaria". Revista Española de Discapacidad, 5 (2): IIII-I 28.

Doi: <https://doi.org/IO. 5569/23405 I04.05.02.06>

Fecha de recepción: I 8-02-20I7 Fecha de aceptación: 22-06-20I7 


\section{Introducción}

La inclusión de todos los alumnos con independencia de sus condiciones personales o ambientales, afecta a la estructura, organización, metodología y actuaciones de los centros educativos. El modo de hallar un consenso entre el sistema escolar y los planteamientos inclusivos planteados por Booth y Ainscow (2002, 20II), puede encontrarse en el análisis y aplicación de las aportaciones derivadas de conceptos como el de calidad de vida (Schalock y Verdugo, 2002, 2003), participación en actividades diversas (King et al., 2003) o desarrollo resiliente (Ungar, 2OI2), desde un enfoque del desarrollo infantil basado en la aplicación de los derechos humanos (ONU, I989).

Estos tres conceptos aluden al logro de estándares relacionados con las condiciones de vida de una persona y la satisfacción que estas condiciones le proporcionan. Implementar estas aportaciones teóricas a la realidad de niños con necesidades específicas de apoyo educativo (NEAE) y sin ellas, requiere un proceso de reflexión sobre las contribuciones de cada concepto a la mejora de la respuesta educativa ofrecida a todo el alumnado.

\section{- Estándares para el crecimiento positivo de todos los niños}

El derecho a la educación de todos los niños, con independencia de cualquier condición personal o ambiental, ha promovido iniciativas y acciones a lo largo de todo el mundo dirigidas desde muy diversos ámbitos: políticos, sociales, educativos, o sanitarios. En esta línea, la UNESCO ha impulsado el programa Escuela para Todos (EPT) que define la educación inclusiva como: Un proceso de abordaje y respuesta a la diversidad de las necesidades de todos los/as alumnos/as a través de la creciente participación en el aprendizaje, las culturas y las comunidades, y de la reducción de la exclusión dentro y desde la educación. Implica cambios y modificaciones en los enfoques, las estructuras, las estrategias, con una visión que incluye a todos los/as niños/as de la franja etaria adecuada y la convicción de que es responsabilidad del sistema regular educar a todos los niños (UNESCO, 2005: I3).

Actualmente, la inclusión es uno de los grandes retos de la educación mundial que conlleva transformaciones cualitativas en el modo de concebir, organizar, planificar, impartir y evaluar la enseñanza y el aprendizaje de las personas en todos los ámbitos socioeducativos (Muntaner et al., 2010). Dado que la inclusión es un fenómeno social antes que educativo, que nos remite a prácticas de actuación democráticas, su implementación en la escuela supone dos procesos interrelacionados. Por un lado, el incremento de la participación de los estudiantes en el currículum de las escuelas ordinarias, por otro, la reducción de la exclusión de los alumnos de las comunidades y culturas de la mayoría (Parrilla, 2002). Algunos de los marcos de referencia que permiten configurar la inclusión en el contexto educativo son: la defensa de los derechos humanos del niño (ONU, I989), la concepción social de discapacidad (AAIDD, 20I I; WHO, 200I; UNESCO, 2006), así como la perspectiva organizativa como base del desarrollo institucional, los modelos de recursos comunitarios y los enfoques de investigación aplicada dirigidos a la acción (Schalock y Verdugo, 2002, 2003; Verdugo y Schalock, 20I3). Desde este prisma, la inclusión educativa se constituye como un indicador de calidad que supone garantizar los principios de igualdad, no discriminación, accesibilidad y diseño universal, guiados por el derecho a la educación de todos los alumnos (Booth y Ainscow, 2002, 20II; Calderón, 20I4; Wehmeyer, 2009).

Estrechamente relacionados con los principios inclusivos, tres modelos conceptuales defendidos por parte de la comunidad científica especializada en crecimiento infantil positivo, están cambiando los modos y formas de abordar las intervenciones educativas, sanitarias y sociales con menores. Por una parte, la percepción que los niños tienen de su calidad de vida y de aquellos aspectos que conforman su bienestar personal, social y emocional. Por 
otra, su participación en actividades diversas, y por último, las características resilientes propias y de su entorno que actúan como fuentes de protección o riesgo ante los desafíos del día a día, influyendo en el crecimiento infantil.

En este trabajo, la calidad de vida se conceptualiza desde el modelo propuesto por Schalock y Verdugo (2002, 2003), centrado en el desarrollo, evaluación y aplicación de indicadores dirigidos a operacionalizar resultados personales relevantes y valiosos para las personas con y sin discapacidad en diferentes áreas de vida (educación, salud, familia, etc.). Tras un extenso trabajo de revisión y construcción científica los autores definen calidad de vida como un estado deseado de bienestar personal, que se conceptualiza a partir de ocho dimensiones (bienestar emocional, relaciones interpersonales, bienestar material, desarrollo personal, bienestar físico, autodeterminación, inclusión social y derechos), que han de contemplarse desde un punto de vista objetivo y subjetivo. Estas dimensiones contienen propiedades tanto universales como relativas a la cultura, y son sensibles a las características personales y a factores ambientales del entorno en el que viven las personas.

La investigación sobre calidad de vida infantil, realizada bajo los planteamientos de Schalock y Verdugo (2002, 2003), ha evidenciado que a la edad de 8 años no se hallan diferencias en la satisfacción que muestran los niños en las distintas dimensiones de vida en función de características personales como la presencia de NEAE (Verdugo y Sabeh, 2002). Sin embargo, en la adolescencia se ponen de manifiesto diferencias en las dimensiones de desarrollo personal, bienestar físico y autodeterminación entre alumnos con NEAE y sin ellas. El grupo de adolescentes con NEAE expresa menores niveles de satisfacción que sus compañeros sin ellas (Gómez-Vela, 2007).

Basándonos en la creciente evidencia científica sobre la relevancia de la participación infantil y juvenil en diferentes áreas de vida, en especial en aquellas destinadas al ocio y disfrute (Badia et al., 2013; King et al., 2007; Majnemer et al.,
20I0), y su relación con el bienestar y calidad de vida de niños y jóvenes con NEAE y sin ellas (Badia et al., 20I3; Schreuer et al., 20I4; Shikako-Thomas et al., 20I2), la participación en actividades de ocio y tiempo libre aparece como otra variable determinante del desarrollo pleno de alumnos con NEAE y sin ellas. En la Clasificación Internacional del Funcionamiento, la Discapacidad y los Estados de Salud (CIF, en sus siglas en español), la participación se conceptualiza como el acto de involucrarse en situaciones de la vida (WHO, 200I). En la CIF para niños y jóvenes (WHO, 2007) las actividades de ocio se definen como el tiempo destinado a actividades elegidas libremente, que los niños realizan cuando no están involucrados en áreas vitales de autocuidado o trabajo escolar.

Dado que el ocio se entiende como una experiencia personal o social caracterizada por la libertad de elección y la experimentación de sentimientos de bienestar, satisfacción, relaciones de amistad y sentido de pertenencia (Hawkins, I993; Imms et al., 2016), los expertos han asociado la participación en actividades lúdicas a resultados personales en calidad de vida. En esta línea, la investigación ha evidenciado que la asistencia a actividades de ocio favorece el bienestar subjetivo del niño y contribuye a la satisfacción en diferentes áreas de vida, tales como el bienestar físico y emocional (DahanOliel et al., 20I 2; Schreuer et al., 20I4), el desarrollo de competencias personales y sociales (Shikako-Thomas et al., 20I2; Wilkes et al., 20I I) y, la autodeterminación cuando se dan oportunidades a los menores para la elección y control de las actividades realizadas (Adolfsson et al., 20I I; Bult et al., 20I4), con independencia de la presencia y tipo de discapacidad (Badia et al., 2013).

Sin embargo, a pesar de la relevancia de la expresión de las preferencias personales para resultados de participación significativos (Bult et al., 20I4), buena parte de la investigación sobre participación infantil coincide en destacar el impacto de factores ambientales, como las elecciones familiares o los programas de actividades extraescolares, sobre los niveles de participación en actividades diversas de ocio 
y tiempo libre (Majnemer et al., 2010). Estos resultados son aún más significativos en niños y jóvenes con discapacidad u otras necesidades específicas de apoyo educativo (King et al., 2007; Badia et al., 2013), los cuales perciben más límites y restricciones ambientales para su participación. Los resultados indican que fomentar un desarrollo sano no solo implica promover características saludables como la participación en actividades diversas, sino también disminuir el efecto de aquellos factores que impiden al niño alcanzar el máximo potencial en los diferentes entornos en los que se desarrolla (King et al., 2003; Rodríguez et al., 2013). En este contexto emerge la tercera variable analizada, por su proximidad conceptual y aplicada a los constructos calidad de vida (Moore et al., 2004; Ungar, 2012) y participación en actividades diversas (ShikakoThomas et al., 201 2; Bøttcher y Dammeyer, 2013): el concepto de resiliencia.

La resiliencia se conceptualiza como un proceso dinámico de adaptación al entorno, capaz de potenciar las fortalezas internas del niño y los factores externos que lo favorecen. Según los expertos, la resiliencia nace de la interacción entre la persona y su ambiente y se dirige al crecimiento positivo y al bienestar significativo del niño en cada situación y etapa de vida (Rutter, 2006; Masten, 2007; Ungar, 20I I). Por ello, la resiliencia hace referencia a la propia persona y a los contextos en los que se encuentran las fuentes que posibilitan la manifestación de sus fortalezas y oportunidades para su adaptación y crecimiento personal (Ungar, 2OI2). Este autor distingue las fortalezas generales de la población y el rol específico que juegan cuando individuos, familias o comunidades están bajo situaciones de estrés concretas o continuas, centrándose en buscar indicadores de resiliencia en las variaciones ambientales que se producen como respuesta positiva a la adversidad desde el nivel individual, familiar, comunitario y cultural, y que proporcionan resultados positivos de cambio (Ungar, 20IO, 20II, 20I2).

En el ámbito del desarrollo infantil, el paradigma de resiliencia se encuentra en construcción actualmente. Brooks (2006) define la resiliencia del niño como la capacidad para lidiar eficazmente con la presión causada por los desafíos cotidianos; recuperarse de decepciones, errores y situaciones adversas; desarrollar objetivos claros y realistas para la resolución de problemas; así como interactuar cómodamente con los demás y tratarse a sí mismo y a los demás con dignidad.

En el ámbito educativo, la resiliencia se plantea como una técnica de intervención que enfatiza el potencial humano (UNESCO, $20 \mathrm{I}_{5}$ ). El desarrollo de la resiliencia de los alumnos es fomentar el conocimiento que tienen de sí mismos, del mundo en el que viven y en el que conviven con otras personas y, dado que la socialización es uno de los aspectos esenciales para la promoción de la resiliencia, los contextos sociales tales como la escuela o las actividades de ocio y tiempo libre se presentan como espacios idóneos para fortalecer las competencias resilientes de niños y jóvenes diversos (Albertín et al., 2013). De esta forma, la participación infantil en actividades lúdicas con independencia de sus características personales o ambientales, puede servir a profesores, psicólogos y otros profesionales del campo de la educación como indicador de salud y funcionamiento positivo y, al mismo tiempo, promover la adquisición de competencias para la vida como la creatividad, el optimismo o las habilidades emocionales y sociales (Shikako-Thomas et al., 20I2), así como fomentar el bienestar personal y social a través de experiencias de control y confianza en uno mismo (Albertín et al., 20I3; Adolfsson, 20II).

En este trabajo se han elegido estas tres variables como indicadores de crecimiento positivo de alumnos con NEAE y sin ellas debido a su interrelación con el desarrollo personal pleno y armónico. Tanto el modelo de calidad de vida (Schalock y Verdugo, 2002, 2003; Verdugo y Schalock, 20I3), como el de participación en actividades diversas (WHO, 200I, 2007; King et al., 2003) y el de desarrollo resiliente (Ungar, 20I0, 20I I, 20I2), surgen como guía y criterio para evaluar las estrategias de mejora que llevan a cabo los profesionales del campo del desarrollo infantil y juvenil. De esta forma, 
los resultados derivados de estas investigaciones permiten formular nuevas propuestas prácticas, que sirven a las escuelas y otros servicios educativos como herramientas para reconocer y responder a las diversas necesidades de niños y jóvenes, atendiendo a su bienestar y capacitación para la vida adulta.

El objetivo de este trabajo es analizar los resultados personales en calidad de vida, participación en actividades diversas de ocio y resiliencia de alumnos de primaria con NEAE y sin ellas, y examinar el peso de algunas variables personales o familiares en dichos resultados, a fin de comprender el papel facilitador o limitador de éstas en su bienestar y desarrollo integral.

\section{Metodología}

\section{- Participantes}

En el estudio participaron I 56 alumnos escolarizados en I4 centros de educación primaria de dos comunidades autónomas de España; 6 centros de Castilla y León y 8 de Andalucía. Siete de los centros educativos eran de titularidad pública y 7 de titularidad concertada. Del total de alumnos participantes, 80 eran alumnos con necesidades específicas de apoyo educativo. La edad media de los I 56 participantes era de I I.04 años. En la Tabla I se exponen algunas de sus características demográficas.
Paralelamente 106 familias, padres, madres o ambos, proporcionaron información demográfica sobre el entorno familiar de los alumnos participantes (Tabla 2).

\begin{tabular}{|c|c|c|c|}
\hline & $\begin{array}{l}\text { Padre } \\
(\mathrm{N}=99)\end{array}$ & $\begin{array}{c}\text { Madre } \\
(\mathrm{N}=105)\end{array}$ & $\begin{array}{c}\mathbf{N} \\
\text { TOTAL }\end{array}$ \\
\hline \multicolumn{4}{|l|}{ Nivel educativo } \\
\hline $\begin{array}{l}\text { Sin estudios } \\
\text { formales }\end{array}$ & 5 & 1 & \\
\hline $\begin{array}{l}\text { Educación } \\
\text { Obligatoria }\end{array}$ & 30 & 28 & \\
\hline Educación Superior & 64 & 74 & \\
\hline \multicolumn{4}{|l|}{ Estatus laboral } \\
\hline Autónomo/a & 26 & 11 & \\
\hline Empleado/a & 53 & 59 & \\
\hline Desempleado/a & 19 & 35 & \\
\hline \multicolumn{4}{|c|}{ Miembros dedicado al cuidado del hijo } \\
\hline Ambos progenitores & & & 47 \\
\hline Madre & & & 37 \\
\hline Padre & & & 4 \\
\hline Otros & & & 1 \\
\hline \multicolumn{4}{|l|}{ Ingresos familiares } \\
\hline $\begin{array}{l}\text { Menos de } 9000 € / \\
\text { anuales }\end{array}$ & & & 23 \\
\hline $\begin{array}{l}\text { De } 9000 \text { a } 17000 € / \\
\text { anuales }\end{array}$ & & & 27 \\
\hline $\begin{array}{l}\text { De } 17000 \text { a } 30000 € / \\
\text { anuales }\end{array}$ & & & 25 \\
\hline $\begin{array}{l}\text { Más de } 30000 € / \\
\text { anuales }\end{array}$ & & & 20 \\
\hline
\end{tabular}

Fuente: elaboración propia.

Tabla 1. Características demográficas alumnos (N=156)
\begin{tabular}{|l|c|c|c|c|}
\hline \multirow{2}{*}{ Género } & \multirow{2}{*}{ Curso } & & \multicolumn{2}{|c|}{ NEAE $^{\mathrm{a}}$} \\
\cline { 3 - 5 } & & $\mathbf{N}^{\circ}$ de alumnos & Con & Sin \\
\hline \multirow{2}{*}{ Niña } & $5^{\circ}$ & 23 & 10 & 13 \\
\cline { 2 - 5 } & $6^{\circ}$ & 40 & 21 & 19 \\
\hline \multirow{2}{*}{ Niño } & $5^{\circ}$ & 58 & 30 & 28 \\
\cline { 2 - 5 } & $6^{\circ}$ & 35 & 19 & 16 \\
\hline
\end{tabular}

${ }^{a}$ Necesidades específicas de apoyo educativo.

Fuente: elaboración propia. 


\section{- Instrumentos}

Los alumnos respondieron a tres cuestionarios.

I. El Cuestionario de Calidad de Vida KIDSCREEN-27 (Ravens-Sieberer et al., 2007). Es una escala de calidad de vida transcultural que permite evaluar el bienestar físico, psicológico y social en niños y jóvenes entre 8 y I 8 años a través de cinco dimensiones (bienestar físico, psicológico, autonomía y relaciones familiares, apoyo social y de los amigos y entorno escolar). La versión española presenta propiedades psicométricamente aceptables (Longo et al., 20I2).

2. El Cuestionario de participación en actividades cotidianas CAPE -versión española- (Longo et al., 20I2). Explora la participación en actividades extraescolares por parte de niños y jóvenes entre 6 y $2 \mathrm{I}$ años, con discapacidad y sin ella. Analiza 55 actividades formales e informales agrupadas en 5 tipos (recreativas, físicas, sociales, basadas en habilidad, de autosuperación). De cada una de ellas recoge la frecuencia de participación, el disfrute que proporciona, con quién realiza el niño la actividad y dónde. El Preferences for Activities of Children PAC (King et al., 2007) es un instrumento complementario al CAPE que evalúa las preferencias de participación de los niños en las actividades evaluadas. La versión castellana se encuentra actualmente en proceso de validación (Badia et al., en prensa).

3. El Cuestionario de Resiliencia CYRM28 (Liebenberg et al., 20I2). Evalúa la resiliencia personal y ambiental de jóvenes entre I 2 y 23 años, a través de tres dimensiones de interacción persona-ambiente: individual, familiar y contextual. Los análisis preliminares del funcionamiento de la escala en población infantil española indican una fiabilidad de 0.90 (Ruiz et al., 20I4).

Finalmente, las familias rellenaron un Listado de indicadores ambientales para la calidad de vida del alumnado, que registra variables del entorno familiar, escolar y comunitario que pueden actuar como barreras o facilitadores en el proceso de inclusión educativa, bienestar y desarrollo positivo de los alumnos.

\section{- Procedimiento}

El acceso a los participantes se realizó a través de I4 centros de educación ordinaria de las comunidades autónomas españolas de Catilla y León y Andalucía. En primer lugar, se envió una carta a los centros en la que se informaba al equipo directivo de los objetivos, el procedimiento y los beneficios de la participación en la investigación y solicitud de su colaboración. Cuando la respuesta de la escuela era afirmativa, se establecía el contacto con las familias de los alumnos de $3^{\text {er }}$ ciclo de primaria. El tutor de cada curso era el encargado de entregar a las familias el formulario de consentimiento informado. Una vez autorizado el estudio por parte del centro educativo y obtenido el consentimiento informado de las familias se procedió a la aplicación de los cuestionarios a estudiantes.

La administración de los instrumentos se llevó a cabo en aulas habilitadas con pizarra digital de los centros educativos participantes. La aplicación de los cuestionarios se realizaba en dos sesiones. En la primera se administraban el cuestionario de calidad de vida y la escala de resiliencia, y en la segunda, el cuestionario de participación en actividades de ocio y tiempo libre. El formato de aplicación dependía del número de alumnos participantes de cada centro y de las necesidades de apoyo específicas de cada alumno. En general, la aplicación fue colectiva en los casos de grupos amplios de alumnos sin NEAE, grupos reducidos de alumnos con NEAE y compañeros sin ellas, y de forma individual con aquellos alumnos que requerían apoyos individualizados para su respuesta autónoma.

Durante las sesiones se exponía a los alumnos de forma sucinta el objetivo de la investigación y se describía la tarea que debían realizar. Además, se enfatizaba el carácter anónimo y confidencial, 
así como la importancia de contestar a todas las preguntas. Para facilitar la comprensión de la tarea a todos los alumnos, en la pizarra digital se proyectaba cada uno de los tres cuestionarios, desde su presentación (instrucciones, formato de respuesta, ejemplos de preguntas y respuestas) hasta cada una de las preguntas o ítems que los componían. Se controló el tamaño y color de la letra, así como las fotografías que acompañaban a cada ítem. Cada uno de los ítems era presentado en una diapositiva, acompañado de imágenes que contextualizaban la pregunta formulada. Los alumnos también disponían de plantillas con las opciones de respuesta escritas, así como la representación pictográfica de cada opción, para aquellos alumnos con dificultades en lectoescritura. Las aplicaciones fueron realizadas por una evaluadora entrenada para guiar la tarea de recogida de información. Esta persona se encargaba de leer las instrucciones, describir los tipos de preguntas y respuestas, y ofrecer turnos de preguntas para aclarar dudas o hacer comentarios al respecto. Todo ello con objeto de facilitar la comprensión y respuesta autónoma de todos los alumnos participantes.

La información familiar se recopiló mediante un cuestionario sociodemográfico que se hacía llegar a las familias a través de la escuela. Era recogido al finalizar las sesiones de evaluación con alumnos.

Tras la aplicación de cuestionarios se inició el análisis de los datos. Se realizaron análisis exploratorios y descriptivos de las puntuaciones, así como pruebas paramétricas y no paramétricas para analizar diferencias estadísticas entre subgrupos. Debido al reducido tamaño muestral, los contrastes se realizaron con un nivel de significación de .05. El análisis de los datos se efectúo con el paquete estadístico SPSS-v2I.

\section{Resultados}

\section{Puntuaciones en resultados personales de los alumnos}

Los participantes obtuvieron puntuaciones medias-altas en Calidad de vida (Tabla 3 ) y Resiliencia (Tabla 4), mientras las puntuaciones medias en Participación fueron moderadas en Diversidad (CAPE) y medias-altas en Preferencia de participación (PAC), para los cinco tipos de actividad examinados (Tabla 5).

Tabla 3. Estadísticos descriptivos de las puntuaciones en Calidad de vida ( $N=156)$

\begin{tabular}{|l|c|c|}
\hline Dominio & Rango & Media (DT) \\
\hline Bienestar físico & $0-100$ & $49.44(10.67)$ \\
\hline Bienestar psicológico & $0-100$ & $49.42(9.53)$ \\
\hline Autonomía y Vida familiar & $0-100$ & $50.64(11.79)$ \\
\hline Apoyo social y Amigos & $0-100$ & $49.61(11.31)$ \\
\hline Entorno escolar & $0-100$ & $50.40(10.18)$ \\
\hline
\end{tabular}

Fuente: elaboración propia.

Tabla 4. Estadísticos descriptivos de las puntuaciones en Resiliencia ( $N=156)$

\begin{tabular}{|l|c|c|}
\hline Dominio & Rango & Media (DT) \\
\hline Resiliencia Total & $0-120$ & $105.57(10.3)$ \\
\hline
\end{tabular}

Fuente: elaboración propia. 


\begin{tabular}{l}
\hline Tabla 5. Estadísticos descriptivos de las puntuaciones en Participación (N=156) \\
\begin{tabular}{|l|c|c|c|c|}
\hline \multirow{2}{*}{ Dominio } & \multicolumn{1}{|c|}{ Diversidad (CAPE) } & \multicolumn{2}{|c|}{ Preferencia (PAC ${ }^{\text {}}$ ) } \\
\cline { 2 - 6 } & Rango & Media (DT) & Rango & Media (DT) \\
\hline Recreativas & $0-12$ & $8.09(2.25)$ & $12-36$ & $29.04(3.67)$ \\
\hline Físicas & $0-13$ & $5.51(2.58)$ & $12-39$ & $31.73(5.00)$ \\
\hline Sociales & $0-10$ & $7.28(2.14)$ & $10-30$ & $26.31(3.41)$ \\
\hline basadas en Habilidad & $0-10$ & $3.14(2.07)$ & $10-30$ & $22.22(4.87)$ \\
\hline de Auto-enriquecimiento & $0-10$ & $5.80(1.93)$ & $10-30$ & $21.82(4.48)$ \\
\hline Escala Total & $0-55$ & $29.94(8.43)$ & $55-165$ & $130.88(17.17)$ \\
\hline
\end{tabular}
\end{tabular}

a Versión española del Cuestionario de participación en actividades cotidianas, CAPE.

b Versión española del Preferences for Activities of Children, PAC.

Fuente: elaboración propia.

Antes de realizar contrastes de diferencias entre-grupos, se comprobaron los supuestos de normalidad y homocedasticidad de la varianza. En aquellos casos en los que se demostró la distribución normal de las puntuaciones, se realizaron pruebas $t$ y ANOVAS para analizar la presencia de diferencias en las puntuaciones obtenidas por los alumnos en función de variables personales (género, edad, presencia o no de NEAE) y ambientales (titularidad del centro; nivel educativo y socioeconómico de las familias; estatus laboral de los padres; y miembros dedicados a la crianza del hijo). En aquellos otros casos en los que no se cumplieron los supuestos de normalidad y potencia estadística, se realizaron pruebas no paramétricas (U de Mann-Whitney y $\mathrm{H}$ de Kruskal-Wallis) para la detección de diferencias entre-grupos.

\section{Puntuaciones en resultados personales en función de variables personales}

En relación con el género de los alumnos, los análisis no evidenciaron diferencias estadísticamente significativas en las puntuaciones de las dimensiones de Calidad de Vida, ni en Resiliencia. Sí se encontraron diferencias significativas en las puntuaciones de Diversidad de participación en actividades de ocio y tiempo libre $\left[\mathrm{t}_{(\mathrm{13})}=-2.40, \mathrm{p}<.05\right]$, pero no en la Preferencia de participación hacia esas mismas actividades. Las niñas puntuaron más alto en Diversidad que los niños [( $M=3$ I.96;
$\mathrm{DT}=7.53)$ vs. $(\mathrm{M}=28.56 ; \mathrm{DT}=8.76)]$. Dada la normalidad de las distribuciones, se efectuaron pruebas $t$ para llevar a cabo estos contrastes.

En función del curso, no se hallaron diferencias significativas en las dimensiones de Calidad de Vida, ni en las puntuaciones en Resiliencia. Pero sí se encontraron diferencias estadísticamente significativas en las puntuaciones de Diversidad y Preferencia de participación en Actividades Recreativas; $(\mathrm{z}=-\mathbf{2} .33, \mathrm{p}<.05)$ y $(\mathrm{z}=-\mathbf{2 . 8 9}, \mathrm{p}<.0 \mathrm{I})$ respectivamente. Los alumnos más pequeños obtuvieron puntuaciones más elevadas en ambos dominios de actividad.

Con respecto a la presencia de NEAE, se hallaron diferencias significativas en la dimensión de Bienestar Emocional de Calidad de Vida $(\mathrm{z}=-2.53, \mathrm{p}<.05)$ y en las puntuaciones en Resiliencia $(\mathrm{z}=-2.55, \mathrm{p}<.05)$. En ambas variables los alumnos con NEAE obtuvieron puntuaciones inferiores al grupo de compañeros sin ellas. No se obtuvieron diferencias significativas en las puntuaciones de Diversidad y Preferencia de participación entre alumnos con y sin NEAE, pero sí en el dominio de Actividades Físicas de Diversidad de Participación ( $\mathrm{z}=-\mathbf{2}$. IO, $\mathrm{p}<.05$ ). Nuevamente los alumnos con NEAE obtuvieron puntuaciones inferiores que sus compañeros sin ellas. La Tabla 6 muestra las puntaciones promedio de aquellos dominios en los que se obtuvieron diferencias significativas entre ambos grupos de alumnos. En estos contrastes, se efectuaron pruebas U de Mann-Whitney al no ajustarse las distribuciones a la normalidad. 
Tabla 6. Puntuaciones promedio de las dimensiones con diferencias significativas entre el grupo con NEAE y sin ellas

\begin{tabular}{|l|l|c|c|c|}
\hline Escala & Subescalas & NEAE $^{\mathbf{a}}$ & $\mathbf{N}$ & Rango $^{\mathbf{b}}$ \\
\hline \multirow{2}{*}{ Calidad de Vida } & \multirow{2}{*}{ Bienestar Emocional } & Con & 77 & 67.62 \\
\cline { 3 - 5 } & & Sin & 75 & 85.62 \\
\hline \multirow{2}{*}{ Resiliencia } & Resiliencia Total & Con & 71 & 61.49 \\
\cline { 3 - 5 } & & Sin & 68 & 78.88 \\
\hline $\begin{array}{l}\text { Diversidad de } \\
\text { Participación }\end{array}$ & Actividades Físicas & Con & 75 & 68.10 \\
\cline { 3 - 5 } & & Sin & 75 & 82.90 \\
\hline
\end{tabular}

a Necesidades específicas de apoyo educativo.

${ }^{\mathrm{b}}$ Rango promedio, prueba U de Mann Whitney.

Fuente: elaboración propia.

\section{Efecto de variables ambientales en resultados personales}

Se analizaron los resultados personales de los alumnos en función de la titularidad del centro educativo. Los alumnos de las escuelas concertadas mostraron valores de Diversidad de Participación significativamente mayores que los alumnos de centros públicos en tres de las cinco tipologías de actividad (Tabla 7), así como en la escala total de Diversidad $\left[\mathrm{t}_{(\mathrm{I} 36)}=-3.82, \mathrm{p}<\right.$ $.00 \mathrm{I}]$. Mientras que no se obtuvieron diferencias entre unos y otros alumnos en los resultados de Calidad de Vida, Resiliencia, o Preferencia de participación en actividades de ocio y tiempo libre.

Cuando se analizaron los resultados personales obtenidos por el grupo de alumnos con NEAE, los datos volvieron a mostrar diferencias significativas en Diversidad de participación en función de la titularidad del centro, pero no en Calidad de Vida, Resiliencia o Preferencia de Participación. De nuevo los alumnos con NEAE de los centros concertados obtuvieron puntuaciones mayores en Diversidad de Participación que sus compañeros con NEAE de centros públicos (Tabla 7 ).

Tabla 7. Resultados en Diversidad de Participación de la muestra total y el subgrupo de alumnos con NEAE en función de la titularidad del centro

\begin{tabular}{|c|c|c|c|c|c|c|c|c|}
\hline \multirow{2}{*}{$\begin{array}{l}\text { Diversidad de } \\
\text { Participación }\end{array}$} & \multirow{2}{*}{$\mathbf{N}^{\circ}$ Act $^{\mathrm{a}}$} & \multirow{2}{*}{$\begin{array}{l}\text { Tipo } \\
\text { centro }^{\mathrm{b}}\end{array}$} & \multicolumn{3}{|c|}{ MUESTRA TOTAL } & \multicolumn{3}{|c|}{$\begin{array}{c}\text { GRUPO DE ALUMNOS } \\
\text { CON NEAE }\end{array}$} \\
\hline & & & $\mathbf{N}$ & Rango $^{c}$ & z & $\mathbf{N}$ & Rango $^{c}$ & $\mathbf{z}$ \\
\hline \multirow{2}{*}{$\begin{array}{l}\text { Actividades } \\
\text { Recreativas }\end{array}$} & \multirow{2}{*}{12} & P. & 73 & 62.53 & \multirow{2}{*}{$-3.16^{\star \star}$} & 38 & 30.79 & \multirow{2}{*}{$2.51^{*}$} \\
\hline & & C. & 73 & 84.47 & & 34 & 42.88 & \\
\hline \multirow{2}{*}{$\begin{array}{l}\text { Actividades } \\
\text { Sociales }\end{array}$} & \multirow{2}{*}{10} & P. & 75 & 63.89 & \multirow{2}{*}{$-2.86^{\star \star}$} & 38 & 31.70 & \multirow{2}{*}{$2.27^{\star}$} \\
\hline & & C. & 71 & 83.65 & & 34 & 41.87 & \\
\hline \multirow{2}{*}{$\begin{array}{l}\text { Actividades de } \\
\text { Habilidad }\end{array}$} & \multirow{2}{*}{10} & P. & 75 & 63.65 & \multirow{2}{*}{$-3.39^{\star \star}$} & 38 & 33.26 & \multirow{2}{*}{-1.18} \\
\hline & & C. & 75 & 87.35 & & 36 & 41.97 & \\
\hline
\end{tabular}

a Número de actividades de cada tipología de actividad valorada por el CAPE.

b Titularidad del centro: P, público; C, concertado.

' Rango promedio, prueba U de Mann Whitney.

"valores significativos $\mathrm{p}<.05$; **valores muy significativos $\mathrm{p}<.0 \mathrm{I}$; ** valores altamente significativos $\mathrm{p}<0.00 \mathrm{I}$.

Fuente: elaboración propia. 
Respecto a las variables del contexto familiar, no se hallaron diferencias significativas en las puntuaciones de los participantes en Calidad de Vida, Resiliencia y Participación en función del nivel educativo de la madre (estudios obligatorios o superiores), pero sí en función de los del padre $(\mathrm{z}=-\mathbf{2 . 2 0}, \mathrm{p}<.05)$. Los contrastes U de Mann-Whitney mostraron puntuaciones promedio más altas en Resiliencia en alumnos con padres con un nivel educativo superior $($ Promedio $=48.59$ vs. Promedio=35.73) . Cuando se realizaron esos mismos análisis con el grupo de alumnos con NEAE, las pruebas $t$ evidenciaron diferencias significativas en las puntuaciones de Preferencia de Participación en Actividades Recreativas en función del nivel educativo de la madre $\left[\mathrm{t}_{(40)}=-2.3 \mathrm{I}, \mathrm{p}<.05\right]$. En este caso, los alumnos con NEAE mostraron puntaciones más elevadas en Preferencia cuando el nivel educativo de la madre era superior.

Los análisis no demostraron diferencias significativas en las puntuaciones de los participantes en ninguna de las variables dependientes analizadas en función del estatus laboral de los padres (autónomo, empleado o desempleado), pero sí con respecto al nivel socioeconómico de las familias (ingresos bajos, medios, medios-altos y altos). En el grupo de alumno con NEAE se encontraron diferencias significativas en función del nivel de ingresos familiares en Diversidad de Participación $\left[\mathrm{F}_{(3,36)}=\right.$ 3.I 8, $\mathrm{p}<.05]$ y Bienestar Escolar $\left[\mathrm{c}_{(3,45)}{ }^{2}=9.05\right.$, $\mathrm{p}<.05]$. Los ANOVAs realizados pusieron de manifiesto que los alumnos con NEAE de familias con niveles de ingresos más altos obtuvieron puntuaciones significativamente mayores en Diversidad de Participación $(\mathrm{M}=34.30 ; \mathrm{DT}=9.9 \mathrm{I})$ que los de familias con ingresos medios $(\mathrm{M}=\mathbf{2 2 . 8 8}$; DT=6.73). Mientras que los análisis no paramétricos para más de dos muestras independientes (prueba KruskalWallis) evidenciaron puntuaciones promedio más altas en Bienestar Escolar en alumnos con NEAE de familias con ingresos bajos (Promedio=31.23), que los de familias con niveles medios (Promedio $=\mathrm{I} 8.50$ ), medios altos $($ Promedio $=\mathrm{I} 7.75)$ y altos $($ Promedio $=24.64)$. Cuando se examinaron los resultados personales del grupo de alumnos sin NEAE en función del nivel de ingresos, se hallaron diferencias significativas en su percepción de Bienestar Emocional $\left[\mathrm{c}_{(3,49)}^{2}=7.9 \mathrm{I}, \mathrm{p}<.05\right]$ y Familiar $[\mathrm{c}$ $\left.{ }_{(3,49)}=\mathrm{I} 4.6 \mathrm{I}, \mathrm{p}<. \mathrm{OI}\right]$. Los alumnos sin NEAE de familias con niveles socioeconómicos medios puntuaban significativamente más alto en Bienestar Emocional y Familiar que sus compañeros de familias con ingresos bajos y altos (Tabla 8). De nuevo, para estos contrastes se efectuaron pruebas Kruskal-Wallis al no ajustarse las distribuciones a la normalidad.

\begin{tabular}{|c|c|c|c|}
\hline & Grupo de alu & s sir & NEAE \\
\hline & $\begin{array}{c}\text { Nivel de } \\
\text { ingresos }^{\mathrm{a}}\end{array}$ & $\mathbf{N}$ & Rango $^{\text {b }}$ \\
\hline Bienestar & Bajos & 10 & 24.00 \\
\hline & Medios & 10 & 27.55 \\
\hline & Medios-Altos & 17 & 30.53 \\
\hline & Altos & 12 & 15.88 \\
\hline Bienestar & Bajos & 10 & 18.45 \\
\hline & Medios & 10 & 27.55 \\
\hline & Medios-Altos & 17 & 34.00 \\
\hline & Altos & 12 & 15.58 \\
\hline $\begin{array}{l}\text { a Bajos (meno } \\
\text { I } 7000 € / \text { año } \\
\text { año); Altos ( } \\
{ }^{b} \text { Rango prom } \\
\text { Fuente: elabo }\end{array}$ & $\begin{array}{l}9000 € / \text { año); } N \\
\text { edios-Altos (de } \\
\text { de } 30000 € / \text { ñno } \\
\text { prueba c c de } \\
\text { on propia. }\end{array}$ & $\begin{array}{c}\text { of } \\
\text { ral- } \\
\text { al }\end{array}$ & $\begin{array}{l}9000 \mathrm{a} \\
0000 € \\
\text { llis. }\end{array}$ \\
\hline
\end{tabular}

Por último, al analizar las puntuaciones obtenidas por los alumnos en función del progenitor ocupado del cuidado y crianza del hijo (ambos progenitores o solo la madre) se hallaron diferencias significativas en la puntación total de Resiliencia, tanto en la muestra total $(\mathrm{z}=-2.36, \mathrm{p}<. \mathrm{OI})$, como en el grupo de alumnos con NEAE ( $\mathrm{z}=-\mathbf{2 . 9 2}, \mathrm{p}<. \mathrm{OI})$, según las pruebas no paramétricas $U$ de Mann-Whitney realizadas. Las puntuaciones fueron superiores cuando ambos progenitores compartían el cuidado y la crianza del hijo (Tabla 9). 


Tabla 9. Puntuaciones promedio obtenidas en
Resiliencia en función del miembro dedicado al
cuidado y crianza del hijo/a
\begin{tabular}{|l|c|c|}
\hline & $\begin{array}{c}\text { Muestra Total } \\
\text { Total de Alumnos } \\
\mathbf{N}=156\end{array}$ & $\begin{array}{c}\text { Grupo } \\
\text { Grupo de } \\
\text { Alumnos con } \\
\text { NEAE } \mathbf{N}=\mathbf{8 0}\end{array}$ \\
\hline $\begin{array}{l}\text { Crianza del } \\
\text { hijo/a }\end{array}$ & Rango & Rango $^{\mathrm{b}}$ \\
\hline $\begin{array}{l}\text { Ambos } \\
\text { progenitores }\end{array}$ & 44.48 & 26.06 \\
\hline Madre & 32.43 & 15.32 \\
\hline
\end{tabular}

${ }^{a}$ Necesidades específicas de apoyo educativo.

${ }^{\mathrm{b}}$ Rango promedio, prueba U de Mann Whitney.

\section{Discusión}

A continuación se discuten los resultados en calidad de vida, resiliencia y participación de los alumnos de primaria y la influencia que las variables personales y ambientales examinadas tienen sobre ellos, a la luz de la actual literatura científica sobre desarrollo infantil y bienestar personal.

\section{Resultados en calidad de vida}

El análisis de los resultados nos permite concluir que los alumnos participantes, en general, tienen una percepción positiva de su calidad de vida, mostrándose satisfechos con las posibilidades para el crecimiento y desarrollo que les brinda su entorno familiar, escolar y de amistad. Estos resultados coinciden con los obtenidos en la investigación transcultural realizada por Erhart y colaboradores (2009) sobre la salud mental y el bienestar de la población infantil europea (Erhart et al., 2009). Las puntuaciones más positivas se refieren a los ámbitos familiar y escolar, lo que pone de manifiesto la importancia del entorno cercano del niño en la percepción que tiene de su bienestar personal, tal y como defiende el modelo de calidad de vida individual de Schalock y Verdugo (2002, 2003).
Sin embargo, pese a los buenos resultados generales en calidad de vida, los contrastes de hipótesis realizados ponen de manifiesto diferencias significativas entre alumnos en las dimensiones de bienestar en función de variables personales y ambientales.

Con respecto a las variables personales analizadas (género, curso y presencia de NEAE), la única variable que ha dado lugar a diferencias en calidad de vida ha sido la presencia de NEAE. En concreto, los alumnos con NEAE participantes en este estudio declaran un bienestar emocional significativamente menor que sus compañeros sin ellas. Estos resultados son de suma relevancia para las trayectorias de desarrollo de unos y otros niños, si tenemos en cuenta los indicadores que definen dicha dimensión: satisfacción, autoconcepto, afectos positivos, espiritualidad y seguridad personal (Verdugo y Schalock, 20I3; Wallander y Koot, 20I6), y su relación con variables del entorno cercano para el logro de valores positivos en bienestar personal (Schalock y Verdugo, 2002, 2003). Además, estos hallazgos son consistentes con los obtenidos por GómezVela (2007) con alumnos adolescentes con NEAE y sin ellas (de I 2 a I 8 años de edad), y ponen en evidencia que las diferencias en bienestar personal pueden también ocurrir a edades preadolescentes, en contraposición a los resultados obtenidos por Verdugo y Sabeh (2002) con alumnos de 8 a I I años de edad, quienes no hallaron diferencias significativas en bienestar emocional entre alumnos con NEAE y sin ellas.

En relación a las variables ambientales, mientras que, menores niveles socioeconómicos se asocian de manera significativa a mayor percepción de bienestar escolar en alumnos con NEAE; en el grupo de alumnos sin NEAE, un mayor estatus socioeconómico familiar se relaciona con niveles significativamente menores de bienestar emocional y bienestar familiar, siendo los niveles socioeconómicos medios los que mayor bienestar emocional y familiar reportan. Estos resultados resaltan nuevamente la importancia del carácter subjetivo del bienestar desde edades tempranas, en contra de aquellas posturas reduccionistas que defienden una vida de calidad desde el acceso y posesión de recursos materiales. Parece 
que para los niños, por delante de la posesión de bienes y recursos económicos se encuentran la cohesión familiar, el desarrollo de relaciones de intimidad y afecto con sus seres queridos, la seguridad de las figuras de apego y la vivencia compartida de experiencias familiares. Por ello, son los niveles económicos intermedios los que permiten responder tanto a las necesidades materiales básicas para la subsistencia de los niños, como a sus necesidades personales y sociales primarias, que alientan el desarrollo personal y la libertad del niño, lo que Amartya Sen (I993, 2002) define como bienestar.

Cuando los niños perciben carencias (físicas, materiales, emocionales, afectivas, entre otras) en sus ambientes cercanos, buscan la respuesta a sus necesidades en otras parcelas. A edades tempranas, como la de los alumnos participantes, las fallas afectivas o socioeconómicas familiares son cubiertas por la institución escolar, donde los niños encuentran personas significativas (iguales, profesionales y otros adultos) que responden y apoyan su crecimiento, así como una estructura organizada que idealmente cubre sus necesidades primarias, amortigua sus dificultades e inseguridades y modela su desarrollo personal y social. Por tanto, la escuela como organización tiene la capacidad de generar en los niños sentimientos de seguridad y bienestar, siendo especialmente significativa para niños en situación de mayor riesgo asociados a su desarrollo, como en este estudio son los alumnos con NEAE de familias con niveles socio-económicos bajos (Ungar, 20II).

\section{Resultados en participación en actividades diversas}

En relación con la participación, la investigación a nivel internacional ha puesto de manifiesto los beneficios en el funcionamiento y bienestar del niño asociados a la realización de actividades de ocio y tiempo libre (WHO, 200I, 2007; King et al., 2003; Badia et al., 2013). En nuestro estudio, los alumnos declaran participar en un número moderado de actividades fuera del horario escolar, aunque muestran claras preferencias por participar en un número mayor de actividades de tipo recreativo, físico y social. Estas actividades se refieren a jugar con las mascotas, ver la TV, ir a pasear, hacer deporte de equipo, jugar a juegos o ir en bicicleta, visitar a otras personas, escuchar música. Estos resultados concuerdan con el informe emitido por el Comité español de UNICEF sobre la infancia en España (20I4), según el cual la inexistencia de documentos, estadísticas o políticas sobre educación no formal provoca una invisibilidad de ésta en el currículo formativo de niños, niñas y jóvenes, limitando su participación en grupos de iguales y su formación integral a actividades desarrolladas en el ámbito de la educación formal.

Con respecto a las variables personales y familiares analizadas, los alumnos con NEAE informan de menores niveles de participación que sus compañeros sin ellas, siendo significativa esta diferencia en la realización de actividades de tipo físico. Este resultado coincide con los de otras investigaciones con niños y jóvenes con discapacidad (Badia et al., 20I3). El hecho de que muchas actividades no estén diseñadas desde planteamientos inclusivos puede encontrarse en la base del mismo, tal y como se expone en el informe sobre los derechos del niño elaborado por el Síndic de Greuges (20I4). No obstante, los alumnos con NEAE manifiestan preferencias claras respecto a su participación en el conjunto de actividades planteadas.

Por otra parte, resulta interesante destacar la influencia que tienen sobre la participación infantil factores ambientales, tales como: el nivel socioeconómico de las familias; la titularidad de la escuela a la que asisten los alumnos (pública o concertada); o el nivel educativo de la madre. La relación hallada entre estas variables y los resultados en diversidad y preferencia de participación apoyan la hipótesis defendida por otros autores, acerca de la relevancia de los factores ambientales sobre los personales en los resultados de participación de niños con discapacidad y sin ella (King et al., 2007; Majnemer et al., 2010).

Más concretamente, en relación con variables de tipo ambiental, los resultados muestran 
mayores índices de participación en actividades de ocio y tiempo libre en alumnos con NEAE de familias con estatus socioeconómicos altos, lo que confiere al nivel socioeconómico un papel facilitador para la asistencia del hijo con NEAE a actividades de ocio diversas (Shikako-Thomas et al., 20I2). Pero como hemos argumentado anteriormente, el nivel socioeconómico de las familias es solo un componente más en el entramado de factores relacionados con el desarrollo del niño. De esta forma, la escuela como agente educativo institucionalizado, adquiere un papel central en las trayectorias de desarrollo de sus alumnos, mediante la movilización efectiva de sus recursos materiales y personales (Booth y Ainscow, 2002, 20II). A este respecto, los resultados personales en diversidad de participación en función de la titularidad del centro obtenidos en esta investigación, indican un mayor fomento de la participación infantil en actividades de ocio por parte de los centros concertados participantes. Lo que se deduce de la información ofrecida por los alumnos de estos centros, los cuales informan de manera sistemática de una mayor y más variada participación en actividades de ocio y tiempo libre que sus compañeros de los centros educativos públicos, con independencia de la presencia de NEAE.

Dadas las evidencias científicas sobre las barreras físicas, materiales y actitudinales hacia la participación de niños y jóvenes con discapacidad en actividades extraescolares de ocio y tiempo libre (Longo y Badia, 2009; King et al., 2009), así como las diferencias en participación halladas entre alumnos con NEAE matriculados en centros escolares específicos y ordinarios (Badia, et al., 20I I), cabe preguntarse por el carácter inclusivo de las actividades ofrecidas por escuelas de diferente titularidad, por las oportunidades creadas para el acceso y participación de todos los alumnos en los programas extraescolares, y por las barreras ambientales para la interacción entre iguales existentes en los centros educativos, que perjudican la asistencia y la participación significativa de los alumnos con NEAE matriculados en escuelas ordinarias (Law et al., 2007).
En definitiva, lo resultados personales en participación destacan la importancia de valorar los factores ambientales que benefician o perjudican la asistencia y realización de actividades de ocio diversas, así como la relevancia de ofrecer a los alumnos la posibilidad de manifestar sus preferencias con objeto de favorecer el desarrollo de competencias necesarias para la conducta autodeterminada (Verdugo y Schalock, 20I3; Majnemer et al., 2010) y los niveles de bienestar (Schreuer et al., 20I4).

\section{Resultados en resiliencia}

En general, los resultados muestran una percepción positiva que el conjunto de alumnos participantes tiene de sus recursos resilientes. No obstante, cabe destacar la menor percepción que muestran el grupo de alumnos con NEAE de sus competencias resilientes personales, así como de las oportunidades ofrecidas por su entorno para su adquisición.

Entre las variables ambientales, el sistema familiar para la crianza y cuidado del hijo parece jugar un rol importante. Los participantes obtienen puntuaciones más elevadas de resiliencia percibida cuando ambos padres comparten la responsabilidad en el cuidado y educación del hijo, que cuando la responsabilidad es solo materna. Este resultado permite identificar otro factor facilitador del desarrollo integral de los niños, más allá de factores obstaculizadores como pueden ser niveles socioeconómicos familiares bajos o barreras escolares para la asistencia y participación significativa de los niños en actividades extraescolares.

De este modo, a pesar de que las puntuaciones generales obtenidas en la escala de Calidad de Vida y Resiliencia son altas, y moderados los valores en Participación, las diferencias halladas entre alumnos con NEAE y sin ellas resaltan un desajuste entre la interacción de niños con NEAE y su entorno cercano (familia, colegio, comunidad, cultura, entre otros sistemas) tal y como destacan otros autores (Gómez- 
Vela, 2007; King et al., 2003; Ungar, 20II). Desde la perspectiva de los actuales modelos de funcionamiento humano este desajuste causa limitaciones en el desarrollo del niño, a partir de las barreras y obstáculos impuestos por el entorno para el fomento de fortalezas y oportunidades que propicien el desarrollo potencial del propio niño.

En suma, las diferencias significativas halladas en calidad de vida, participación y resiliencia en niños con NEAE y sin ellas, acentúan la urgente necesidad de incluir a aquellos alumnos con NEAE en los diferentes ámbitos sociales donde se dan las oportunidades a los niños de experimentar y tener éxito en su proceso de desarrollo. Dada la naturaleza de la participación en actividades de ocio, ésta se presenta como una estrategia formativa útil para el sistema educativo, capaz de ofrecer respuestas adecuadas a alumnos con NEAE y sin ellas, y posibilitar situaciones para su aprendizaje y logro de resultados personales significativos. Para ello, resulta imprescindible:

I) tener en cuenta las preferencias infantiles para la implementación de diversas actividades de ocio y tiempo libre; 2) adaptar las actividades extraescolares para garantizar el acceso de los alumnos; 3) y aumentar la disponibilidad de las actividades para facilitar la participación de un mayor número de alumnado diverso (Bult et al., 20I4; Engel-Yeger y HannaKasis, 20Iо; Wilkes et al., 20II). Por tanto, la manera de implementar la participación de niños con NEAE y sin ellas en actividades de ocio diversas, es mediante la provisión de apoyos individualizados que favorezcan el acceso y disponibilidad de todos los recursos y oportunidades existentes en el entorno próximo, y promueven la asistencia y desempeño óptimo de todos los niños con independencia de las características personales y variables ambientales presentes en el entorno de cada niño (Booth y Ainscow, 2002, 20I I; Bøttcher y Dammeyer 2013; Wehmeyer, 2009).

\section{Conclusiones}

Como se evidencia en los resultados expuestos la escuela es valorada por los alumnos como un contexto significativo que puede generar y garantizar su bienestar. Pero a nivel social, la escuela es algo más que un simple facilitador del desarrollo positivo del niño, es también un agente de cambio (Schalock y Verdugo, 2002, 2003; Verdugo y Schalock, 2013) capaz de crear oportunidades tales como el fomento de la participación de los alumnos en actividades diversas y preferidas para ellos, que favorecen el desarrollo de fortalezas personales necesarias para el desarrollo integral de los niños, lo que en este estudio se ha conceptualizado como resiliencia (Ungar 20I I), y la calidad en diferentes condiciones de vida. Como ejemplo de claves de cambio educativo citaremos, el análisis del abanico de actividades de ocio disponibles en la escuela y el barrio, la valoración de las preferencias de participación de los alumnos, el examen de las actividades disponibles para comprobar hasta qué punto permiten el acceso, la participación y responden a los intereses y preferencias de los niños, el impulso de apoyos individualizados elaborados a partir de las características del niño, superando los programas educativas formales e informales basados en etiquetas como la de NEAE (Wehmeyer, 2009), así como en el diseño universal para el aprendizaje dirigido a la promoción de la asistencia, participación y aprendizaje significativo de todos los alumnos (Booth y Ainscow, 2002, 201I).

El modelo de participación inclusiva presentado en este trabajo defiende la participación en contextos lúdicos y de ocio como un factor protector capaz de mitigar los riesgos comportamentales y psicopatológicos que nacen de la incongruencia entre el desarrollo personal del niño con NEAE, y las demandas y expectativas de los entornos en los que participa (Bøttcher y Dammeyer, 20I3). El modelo se enfoca a la promoción de factores personales y ambientales con capacidad de apoyar y facilitar resultados personales 
significativos, como en este estudio han sido las labores de crianza compartida por ambos padres o la titularidad del centro educativo. Sin embargo, no hay que perder de vista que fomentar un desarrollo sano no solo implica promover características saludables, sino también disminuir aquellos factores que impiden al niño alcanzar el máximo potencial dentro de cada fase de desarrollo. En este sentido, no solo las familias o centros educativos tienen un papel central en la prevención de riesgos y fomento de oportunidades para el desarrollo saludable y satisfactorio de la infancia, sino también los poderes públicos y administraciones encargadas de implementar las políticas infantiles a nivel local (Ungar, 20I I, 20I2). Especialmente en aquellos casos o colectivos que por características propias o de su ambiente ven limitadas sus oportunidades para exponerse a situaciones sociales diversas que reportan disfrute, bienestar y aprendizaje.

Por tanto, desde una perspectiva ecológica, el cambio debe ir orientado a la interacción de las actuaciones realizadas desde los distintos niveles educativos implicados en el desarrollo infantil. Es decir, a nivel individual, a través del desarrollo de prácticas educativas basadas en los resultados personales de calidad para el alumno e implementación de apoyos individualizados; a nivel de la estructura y función escolar, mediante la mejora de los espacios físicos del centro, cambios organizativos en los equipos docentes, o evaluación y mejora de los programas de actividades extraescolares; y a nivel del macrosistema, referente a la implicación de las políticas públicas y administraciones educativas, a la aplicación de acciones estratégicas orientadas a la promoción del desarrollo positivo, calidad de vida de todo el alumnado, y eficacia de la escuela como organización educativa (Verdugo y Schalock, 2013).

Para concluir, este estudio ha permitido identificar resultados personales del alumnado de primaria, así como variables personales (presencia de NEAE) y ambientales (titularidad del centro educativo; nivel socioeconómico familiar; función familiar para la crianza y cuidado del hijo, por ejemplo) relacionados con ellos. Estos resultados proporcionan evidencia científica de utilidad práctica para el actual proceso de construcción de la escuela inclusiva, a partir de la aplicación del modelo de calidad de vida individual como base práctica para el desarrollo y bienestar de todos los alumnos (Ossa et al., 20I 5). A partir del presente trabajo hemos tratado de aportar fundamentos teóricos y empíricos para el desarrollo de indicadores y estándares que sirvan de herramienta guía para el cambio organizacional de la escuela (Schalock y Verdugo, 2002, 2003; Verdugo y Schalock, 20I3).

Esta aportación científica se suma a la de otros colegas (Gómez-Vela, 2007; Muntaner et al., 2010) comprometidos con la reflexión y creación de prácticas educativas alternativas al currículo educativo oficial. Prácticas educativas basadas en la evidencia, que contribuyan a la mejora de la calidad de la educación, y al logro de una escuela de todos y para todos (Booth et al., 2002, 20I I). No obstante, los resultados presentados son preliminares por lo que se recomienda interpretarlos con cautela, teniendo en cuenta el reducida tamaño muestra con el que se han realizado los contrastes de hipótesis, los cuales en su mayoría han sido efectuados mediante pruebas no paramétricas al no cumplirse los supuestos de normalidad para la comparación de grupos muestrales. Sin embargo, más allá de las limitaciones mencionadas, se alienta a la comunidad científica dedicada a la investigación aplicada en discapacidad, educación y desarrollo infantil positivo a dirigir su foco de atención en investigación al marco teórico de referencia planteado en el actual trabajo, basado en un modelo biopsicosocial de desarrollo, bajo el prisma de los derechos, igualdad y libertad humana. Así como un llamamiento a los profesionales del campo educativo para que reflexionen e implementen métodos de evaluación centrados en la consecución de resultados personales significativos para el alumno, que guíen las prácticas educativas hacia la inclusión y el desarrollo positivo de todo el alumnado. 


\section{Referencias bibliográficas}

Adolfsson, M. et al. (20I I): "Identifying child functioning from an ICF-CY perspective: Everyday life situations explored in measures of participation”. Disability and Rehabilitation, 33 (I3-I4): I230-I 244 .

Albertín, M. R. et al. (2013): "PositiviES: Recursos de la psicología positiva para la educación intra e interpersonal en las escuelas", en Pérez, F. M. (ed.): Variables psicológicas y educativas para la intervención en el ámbito escolar, Almería: Asociación Universitaria de Educación y Psicología.

American Association on Intellectual and Developmental Disabilities, AAIDD (20II): Discapacidad intelectual: definición, clasificación y sistemas de apoyo ( I I ed.) [Traducción de M. A. Verdugo]. Madrid: Alianza Editorial.

Badia M. et al. (20I I): "Personal factors and perceived barriers to participation in leisure activities for young and adults with developmental disabilities". Research in Developmental Disabilities, 32 (6): 205 5-2063.

Badia, M. et al. (20I3): "The influence of participation in leisure activities on quality of life in Spanish children and adolescents with Cerebral Palsy". Research in Developmental Disabilities, 34: 2864-287I.

Booth, T. y Ainscow, M. (2002): Index for inclusion: developing leaning and participation in schools (2nd ed.). Manchester: Centre for Studies in Inclusive Education (CSIE).

Booth, T. y Ainscow, M. (20I I): Index for Inclusion: developing learning and participation in schools ( $3 \mathrm{rd} \mathrm{ed}$.). Bristol: Centre for Studies in Inclusive Education (CSIE).

Bøttcher, L. y Dammeyer, J. (20I3): “Disability as a risk factor? Development of psychopathology in children with disabilities". Research in Developmental Disabilities, 34: 3607-36I7.

Brooks, J. E. (2006): “Strengthening resilience in children and youths: Maximizing opportunities in the schools". Children and Schools, 28 (2): 69-76.

Bult, M. et al. (20I4): "Do children participate in the activities they prefer? A comparison of children and youth with and without physical disabilities”. Clinical Rehabilitation, 28 (4): 388-396.

Calderón, I. et al. (20I4): "Expresión e inclusión. Entrevista a Ignacio Calderón Almendros. La discapacidad no es una realidad biológica y personal, sino que fundamentalmente es de naturaleza social y cultural”. Aularia, 3 (2): 43-46.

Dahan-Oliel, N. et al. (20I2): "Quality of life and leisure participation in children with neurodevelopmental disabilities: a thematic analysis of the literature". Quality of Life Research: An International Journal of Quality of Life Aspects of Treatment, Care and Rehabilitation, 2I (3): 427-439.

Engel-Yeger, B. y Hanna-Kasis, A. (2010): “The relationship between Developmental Coordination Disorders, child's perceived selfefficacy and preference to participate in daily activities". Child Care Health Development, 36 (5): 670-677.

Erhart, M. et al. (2009): "Measuring mental health and well-being of school-children in I 5 European countries using the KIDSCREEN-Io Index". International Journal of Public Health, 54 (2): I $60-166$

Gómez-Vela, M. (2007): "La calidad de vida de alumnos con necesidades educativas especiales y sin ellas. Elaboración de un marco conceptual y un instrumento de evaluación”. Educación y Diversidad, I: I I3-I35.

Hawkins, B. A. (I993): "Leisure participation and life satisfaction of older adults with mental retardation and Down syndrome", en E. Sutton et al.: Older adults with developmental disabilities: optimizing choice and change. Baltimore: Paul H. Brookers Publishing Co. 
Imms, C. et al. (2016): “A descriptive study of the participation of children and adolescents in activities outside school”. BMC Pediatrics, 84 (I6).

King, G. et al. (2003): "A conceptual model of the factors affecting the recreation and leisure participation of children with disabilities". Physical and Occupational Therapy in Pediatrics, 23 (I): 63-90.

King, G. et al. (2007): “Measuring children's participation in recreation and leisure activities: Construct validation of the CAPE and PAC". Child: Care, Health and Development, 33: 2839.

King, G. et al. (2009): “Un modelo conceptual de los factores que afectan a la participación en las actividades de ocio y recreo de los niños con discapacidades”. Siglo Cero, 40 (2): 5-29.

Law, M. et al. (2007): "Perceived environmental barriers to recreational, community, and school participation for children and youth with physical disabilities". Archives of Physical Medicine \& Rehabilitation, 88 (I 2): I636I642.

Liebenberg, L. et al. (2012): "Validation of the Child and Youth Resilience Measure-28 (CYRM-28) among Canadian Youth”. Research on Social Work Practice, 22 (2): 219226.

Longo, E. et al. (2OI2): “Cross-cultural validation of the Children's Assessment of Participation and Enjoyment (CAPE) in Spain”. Child: Care, Health and Development, 40 (2): 23I-24I.

Longo, E. y Badia, M. (2009): “La participación en las actividades de ocio en los niños y adolescentes con parálisis cerebral”. Siglo Cero, $40(4): 79-93$.

Majnemer, A. et al. (2010): "Leisure activity preferences for 6-to I 2-year-old children with cerebral palsy". Developmental Medicine and Child Neurology, 52: I67-I73.

Masten, A. S. (2007): "Resilience and developing systems: Progress and promise as the fourth wave rises. Development and Psychopathology, I9: 92 I-930.
Moore, K. A. et al. (2004): "Indicators of child well-being: The promise for positive youth development". Annals of the American Academy of Political and Social Science, 59I: I25-I45.

Muntaner et al. (2010): Estándares e indicadores para analizar la calidad de vida del alumnado con discapacidad durante su proceso educativo. Palma de Mallorca: Edicions UIB.

Organización de las Naciones Unidas ( I989): Convención sobre los Derechos del Niño (en línea). <http://www.ohchr.org/SP/ ProfessionalInterest/Pages/CRC.aspx $>$, acceso I 5 de febrero de 2017.

Ossa, C. et al. (20I 5): "Construyendo un modelo de calidad de vida escolar", en Saavedra $e t$ al. (ed.): Resiliencia y calidad de vida. La Psicología Educacional en diálogo con otras disciplinas. Talca: Universidad Católica del Maule.

Parrilla, A. (2002): "Acerca del origen y sentido de la educación inclusiva”. Revista de Educación, 327: I I-32.

Ravens-Sieberer, U. et al. (2007): “The KIDSCREEN-27 quality of life measure for children and adolescents: psychometric results from a cross-cultural survey in I 3 European countries". Quality of Life Research, I6 (8): I347-I356.

Rodríguez, G. et al. (2013): "Propuesta de estudio sobre la influencia del estilo educativo parental en el nivel de adaptación e inteligencia emocional de menores en riesgo social”, en Pérez, F. M. (ed.): Variables psicológicas y educativas para la intervención en el ámbito escolar. Almería: Asociación Universitaria de Educación y Psicología.

Ruiz et al. (20I4): "Determinantes de la calidad de vida del alumnado de Primaria". Revista iberoamericana de educación, 66: 59-74.

Rutter, M. (2006): "Implications of Resilience Concepts for Scientific Understanding”. Annals of the New York Academy of Sciences, I094 (I): I-I 2 .

Schalock, R. L. y Verdugo, M.A. (2002): Handbook on quality of life for human service 
Practitioners. Washington, DC: American Association on Mental Retardation.

Schalock, R.L. y Verdugo, M.A. (2003): Calidad de Vida. Manual para profesionales de la educación, salud y servicios sociales. Madrid: Alianza.

Schreuer, N. et al. (2OI4): "Participation in leisure activities: Differences between children with and without physical disabilities". Research in Developmental Disabilities, 35 (I): 223-33.

Sen, A. (I993): “Capability and Well-being”, en M. Nussbaum y A. Sen (eds.): The Quality of Life. Oxford, England: Clarendon Press. [Trad. de R. Reyes, en Nussbaum y Sen (eds.): La calidad de vida (3rd reimpr.). México: Fondo de Cultura Económica, 2002].

Shikako-Thomas, K. K. et al. (20I2): "Play and Be Happy? Leisure Participation and Quality of Life in School-Aged Children with Cerebral Palsy". International Journal of Pediatrics, 387280 (2012)

Síndic de Greuges (20I4): Informe sobre los derechos del niño. 25 años de la convención: principales retos pendientes (en linea). <http:// www.sindic.cat/site/unitFiles/3743/Informe \% 20 infancia \% 20201 $4 \%$ 20castella.pdf $>$, acceso I 3 de diciembre de 2017 .

UNESCO (2005): Guidelines for inclusion: ensuring access to education for all. París: UNESCO.

UNESCO (2006): Convención internacional sobre los derechos de las personas con discapacidad (en línea).

< http://www.un.org/esa/socdev/enable/documents/ tccconvs.pdf $>$, acceso 29 de febrero de 2017.

UNESCO (2015): Informe de seguimiento de la EPT en el mundo, 2015: el desarrollo sostenible comienza por la educación. París: UNESCO.

Ungar, M. (2010): "What is resilience across cultures and contexts? Advances to the theory of positive development among individuals and families under stress". Journal of Family Psychotherapy, 2I (I): I-I6.

Ungar, M. (20II): “The social ecology of resilience: Addressing contextual and cultural ambiguity of a nascent construct". American Journal of Orthopsychiatry, 8I (I): I-I7.

Ungar, M. (20I2): "Social ecologies and their contribution to resilience", en Ungar. M. (ed.): The social ecology of resiliencie: a handbook of theory and practice. New York, NY: Springer.

UNICEF Comité Español (20I4): La infancia en España 20I4. El valor social de los niños: hacia un Pacto de Estado por la Infancia. Madrid: UNICEF.

Verdugo, M. A. y Sabeh, E. N. (2002): "Evaluación de la percepción de calidad de vida en la infancia”. Psicothema, I4 (I): 86-9I.

Verdugo, M.A. y Schalock, R. (eds.) (2013): Discapacidad e Inclusión: manual para la docencia. Salamanca: Amarú Ediciones.

Wallander, J.L. y Koot, H.M. (2016): “Quality of life in children: A critical examination of concepts, approaches, issues, and future directions”. Clinical Psychology Review, 45: I 3 I-I 45 .

Wehmeyer, M.L. (2009): “Autodeterminación y la tercera generación de prácticas de inclusión”. Revista de educación, I49: 45-67.

Wilkes, S. et al. (20I I): “A play-based intervention for children with ADHD: A pilot study". Australian Occupational Therapy Journal, 58 (4): $23 \mathrm{I}-240$.

World Health Organization, WHO (2001): The International Classification Functioning, Disability and Health. Geneva: WHO.

World Health Organization, WHO (2007): The International Classification Functioning, Disability and Health. Children and young. Geneva: WHO. 